\title{
Comparative Verification of Design Ordering Methods using Reader's Feeling for Document Production Out- sourcing Services
}

\author{
Kazuo Shibuta ${ }^{* \dagger}$, Kunihiko Higa *
}

\begin{abstract}
We showed that there are three levels of production purpose, reader's feeling and design specifically as an ordering method when requesting design work. It was statistically confirmed that the appropriateness and the easiness of understanding improve when the order content includes reader's feeling. In particular, it was recognized that appropriateness would be improved if the reader's feeling instruction was given without the production purpose instruction. Furthermore, appropriateness decreased when instructing production purpose and design at the same time. As a result, it is considered that the inclusion of reader's feeling in the content of the order has improved the evalu-ation of the worker for the ordering method.
\end{abstract}

Keywords: design, outsourcing, crowdsourcing, ordering method

\section{Introduction}

\subsection{Document production outsourcing services}

In recent years, as the simple copier business falls into price competition, the industry of office automation equipment has started to provide strategic outsourcing services for offices as a new business to improve profitability. For example, at Fuji Xerox [1], the mainstream is the service that manages the optimal output environment in the office, which is called managed print service. Furthermore, they launched a new service: an outsourcing service for document production, not for managing output environment. With this service, they undertake production of documents used in corporate activities, such as educations and proposals, for example: catalogs, instruction manuals (user's manuals), service manuals, flyers, provisions, etc. for products or services, with optimizing quality, cost, and delivery date. In this service, they undertake not only simple outputs, but also the production of documents including design. It is important to develop operation technologies and systems in general production including design, and to have know-how to product documents.

* Tokyo Institute of Technology, Tokyo, Japan

$\dagger$ Kwansei Gakuin University, Hyogo, Japan 


\subsection{Emergence of crowdsourcing}

On the other hand, along with the development of ICT, the use of crowdsourcing which utilizes ICT and is a new system of human resource procurement has been increasing. "Crowdsourcing" is a coined word that combines "crowd" as a group of people and "sourcing" as procurement, which is said to be used for the first time in a blog article [2] posted on U.S. "WIRED" by Jeff Howe: the contributor to the magazine. It is a system that accesses an unspecified number of individuals or companies using ICT to procure human resources in need. Ordering, application, decision of worker, delivery and payment are done via ICT. Herewith, it became possible to place an order to general individuals such as students, housewives, retired seniors, etc. The contents of jobs are wide-ranging such as development, design, writing, office work, business support, etc. According to the survey by Y's STAFF [3], the jobs ordered in Japan are mostly related to design, web design, web development, etc., and it seems that many clients have needs for special skills.

The jobs will be generally classified into project type, competition type and task type depending on the appointment method of workers and procedure. The project type is a project-based job with a fixed production period and deliverables, and the fee may be decided by negotiation after the worker is appointed. The competition type is a job in which only an outstanding result is adopted, and the fee is often predetermined. The task type is a finely divided task that anyone can do, and the fee is often predetermined. In the cases related to design, it is usual to order in the competition type, such as production of logos, flyers, posters, etc., but there are cases that workers who have been checked the result in the prior competition type may be asked more complex and advanced tasks in the project type.

\section{Purpose of Research}

\subsection{Problems in ordering jobs related to design}

When considering the commercialization of outsourcing services, the current problems of crowdsourcing could be helpful. Although it is not a face-to-face process, an ordering in crowdsourcing is a process of contract, and therefore communication skills of both clients and workers are also required. There are also a few cases where troubles occur over the deliverables. The cause is, for example, that the delivery date is not suitable, but the followings can be considered especially in jobs related to design.

\section{1) Client's instructions on design are complicated}

2) The image for the deliverable is different between the client and the worker

3) The fee for deliverable is underestimated by the client

The ordering process in crowdsourcing includes client ordering, worker's application, worker selection by client, worker's work, and client evaluation of deliverables. Regarding ordering by clients, Kittur et al. [4] explained about a complex job, if the task of creating a long article is divided into small units and requested, the quality of the deliverable was 
effected by the human capability in the middle. In task design, Chandler and Kapelner [5] examined how to manage worker motivation, and Eickhoff and Vries [6] examined how to prevent fraud. Regarding the selection of workers by clients, Kittur et al. [7] showed that incorporating a mechanism to confirm that the instructions were read to the end when placing an order was effective in eliminating workers who answer incorrectly. Regarding the evaluation of deliverables by clients, Heer and Bostock [8] visualized evaluations using "Amazon Mechanical Turk".

In jobs related to design, client's instructions are given in different levels: sometimes in view of the production purpose such as concept, targeted persons, etc., sometimes in specific design such as finished image, coloring, font size, etc. The instructions at these different levels may not be consistent at clients. In addition, because of the difficulty to express everything in words if there is no reference or base, clients may look at the unfinished product to add some instructions. This is the reason that situations like 1) are considered to occur. Furthermore, when the client cannot express the image specifically and gives instructions only for the simple purpose of the product, such as improvement of the purchase rate, the worker cannot have the exact image of the client, and therefore cause troubles. This is the reason that situations like 2) are considered to occur. In Lancers [9], a comprehensive crowdsourcing website in Japan, and CrowdWorks [10], another comprehensive crowdsourcing site, the following ideas have been made for the jobs related to design. At the time of order, they are preparing a template to describe the purpose and outline of production, a user interface to instruct images with a scale such as monotone-colorful, simple-complex, casual-classic, reasonable-high class, traditional-modern, feminine-masculine, or a user interface to select the desired color with a radio buttons such as red, yellow, green, blue, pink, orange, black, etc. Herewith, it is considered that they are trying to solve the problem 2). Furthermore, a contradictory instruction may be given by simultaneously designating a desired image and a desired color. In fact, in the indications of desired color, words related to the image such as bluish (cool, refreshing, masculine), pink (cute, young, feminine), black (dainty, luxury, elegance), etc. are also presented. At last, the value of the product seems to be how much it can achieve its purpose, but it is difficult to predict in advance. That is because it is determined by the reader who is the targeted persons of the product, and not by the client or the worker. Therefore, the worker cannot claim its value objectively to the client. This is the reason that situations like 3) are considered to occur. This also results in the question of whether or not a production should be done faithfully to the client's image regarding the problem 2). These situations can be considered even in the case of regular outsourcing but expected to be particularly noticeable in crowdsourcing because the orders often proceed via ICT.

\subsection{Consideration of an effective ordering method of design}

With regard to such jobs related to design, an effective ordering method using crowdsourcing will be proposed and conduct an experiment to verify the effect. The expected effect will be that the complexity of instructions is eliminated, the image for the product is consistent between the clients and the workers, and the clients don't underestimate the fee for the deliverables. However, it is not considered whether the images of the deliverables match and whether the fee for the deliverables are appropriate at this time, because it is necessary to evaluate the design of the deliverable itself. The evaluation of the worker, in other words the designer, is used as an alternative indicator of the effect. 


\section{Extraction of Problems in Design Ordering}

\subsection{Contents}

In "Promotion program of utilizing crowdsourcing at The Small and Medium Enterprise Agency 2016" [11], with the purpose of promoting crowdsourcing in small and medium enterprises, a crowdsourcing producer offered a program to let participant's experience actual ordering and to raise awareness of crowdsourcing. The problems upon its use were also extracted. The crowdsourcing producer is a company that is certified by the crowdsourcing association under the certification system established in the previous year to support small and medium enterprises with the use of crowdsourcing. This project aims to gain the competitiveness of small and medium enterprises by utilizing crowdsourcing, and it is conducted by the crowdsourcing utilization consortium consists of 3 members, Fuji Xerox Co., Ltd., Crowdsourcing Association in Japan, and Japan Telework Association by the commission of The Small and Medium Enterprise Agency in Japan. In the order experience program, two courses were held. The overview of crowdsourcing was explained in the first round and they registered on the crowdsourcing website and order a flyer design (A4 size) in the competition type. The second round was held about 1 or 2 weeks later, in which they selected from the flyers gathered in the actual competition. The order experience program was held at about 150 locations nationwide in Japan from February to June 2016, with more than 800 small and medium enterprises participated.

\subsection{Research method}

Survey questionnaires about satisfaction levels and problems of small and medium enterprises will be conducted for in the order experience program participants. The satisfaction level is measured by a Likert scale (5: very good, 4: good, 3: neutral, 2: bad, 2: very bad) regarding the entire program, the fee and quality of the deliverables, the operation and registration on the website, etc. Other than that, the definition of requirement (1: well accepted without review, 2: well accepted after reviewed once, 3: well accepted after reviewed for multiple times, 4 : not accepted), the number of applicants or proposals, etc.

\subsection{Result}

The number of valid answers was 733. First, we looked at the relationship between the average satisfaction level of the entire order experience program and whether the requirements were defined successfully. As a result, the satisfaction level decreased when the definition was "not accepted" than else. Through one-way analysis of variance, statistically significant difference $(\mathrm{F}(3,729)=19.14, \mathrm{p}<0.01)$ was indicated (see Figure 1$)$. The error bars in the figure indicates standard errors. 


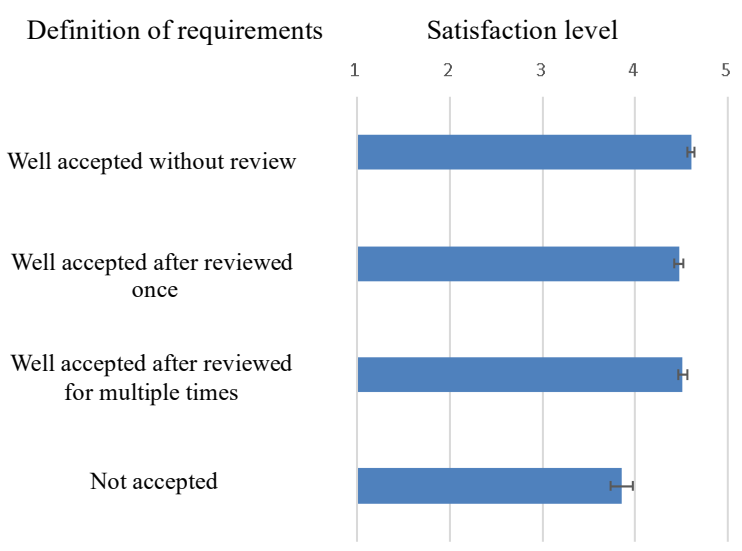

Figure 1: Relationship between satisfaction level and definition of requirements

Next, we look at the relationship between the average number of applicants and proposals, and whether the requirements were defined successfully. As a result, the number of applicants and proposals tend to increase when the definition is well accepted (see Figure 2). The error bars in the figure indicates standard errors.

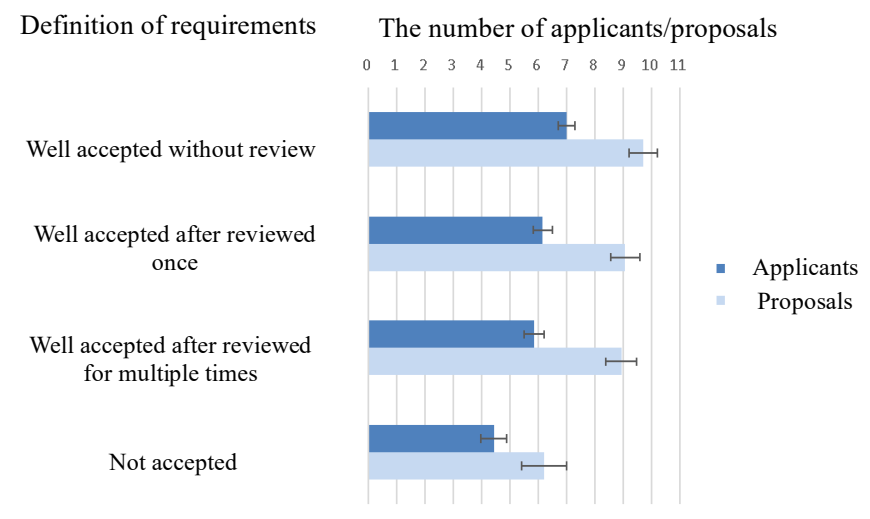

Figure 2: Relationship between

The number of applicants/proposals and definition of requirements

From the analysis results, it was found that both the number of applicants and proposals increase when the definition of requirement is well done at the time of ordering. This is likely to increase satisfaction with the order experience program, as it offers more options for proposals and allows more desirable designs to be adopted.

\section{Consideration of Design Orderings}

\subsection{Design process}

Considering the general design process, this time there is a third party, the reader, in addition to the client and worker. In Axiomatic Design [12], the design process is divided into a customer domain, a functional domain, an actual domain, and a process 
domain, and the customer who is the reader is considered. Further, it is shown that the designer starts with the function requirement of the functional domain. In this case, we will examine what the function requirement is. Since the document design influences the affection, the demand varies greatly from person to person. Matsuoka et al. [13] and Yanagisawa et al. [14] have proposed design models that deal with affection. According to them, customer's psychological factors are defined in the function requirement.

\subsection{Relationship between production purpose and design}

First of all, the final evaluation of the product can be determined by how much it achieved the production purpose. For example: in the case of a catalog, the purchase rate should be improved; in the case of a manual, the understanding level should be improved; in the case of a form, entry errors should be decreased; Furthermore, the satisfaction level of the targeted persons should be increased. However, it is very difficult to objectively show them in advance. The reason may be the fact that how design can be achieved by designing the layout and coloring largely differs depending on the type of the target product and persons. For this reason, in the production work including design, regardless of how the production purpose can be achieved, the fee is usually calculated by multiplying man-hour and the unit price per hour. However, in this case, the reduction in man-hour and the unit price per hour becomes a competitive advantage. On the contrary, there is also a problem that it becomes difficult to design effectively for the production purpose.

On the other hand, in the field of usability evaluation, the following three are often measured: production purpose as whole evaluation, feelings as psychological factor, and design as physical factor (see Figure 3). The psychological factors are, in particular, psychophysical quantities that quantitatively express the reader's feelings such as "easy to understand", "easy to fill in", "reliable", "neat", "fun", etc.

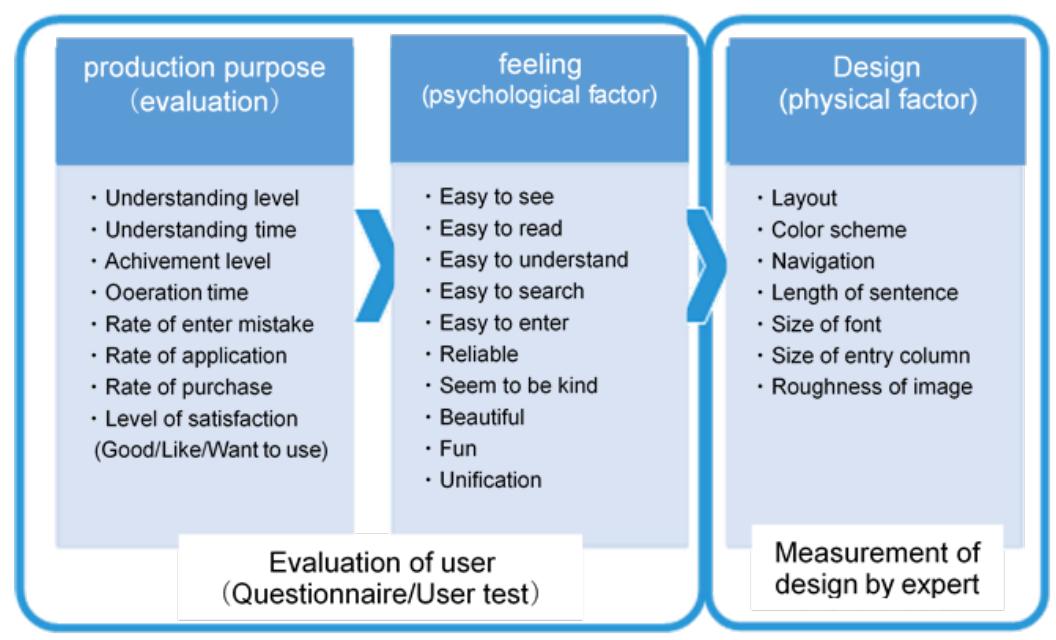

Figure 3: Relationships between production purpose -feeling -design in the usability evaluation

Tanaka et al. [15] classified these psychological factors into several evaluation scales related to journal articles, and then performed multiple regression analysis between the evaluation scales and the final rating "good" and finally indicated that it leads to the regression equation. However, this regression equation is limited to the one objected to journal articles. 
Since the relationship between the psychological factors and the design is related to human perception and cognition as a living body, it is considered that the relation is not greatly influenced by the type of the product and the targeted persons. On the other hand, the relationship between the production purpose and the psychological factors is related to advanced information processing, because it is about what kind of effect do those perceived from the product give human behavior. Therefore, it is considered that this is greatly influenced by the type of product and the targeted persons. In the elaboration likelihood model (ELM) [16], the information processing route for causing attitude change differs depending on the information processing ability and motivation of the consumer; the central route is reported as it uses more direct and logical information, and the peripheral routes are reported as they use rather indirect and sensory information. For example, in the case of a product catalog, some people may be motivated to purchase because of "easy-tounderstand" catalog that explains the details of the product, and some people may prefer an "interesting" catalog that gives an image of the scene using the product. This also varies depending on the product type. The psychological factors required for product catalogs used in business and those used for an individual are expected to be different. This relationship is also influenced by the product type. For example, in the case of a service manual, it is considered that accurate and "reliable" information is written in an "easyto-find" manner to achieve the production purpose. Usually, design patterns are infinite, and it is impossible to directly obtain the relationship between the production purpose and design for each type of product or targeted persons. However, in case of a psychological factor, it is possible to condense it to a statistically finite number using factor analysis, etc. In other words, in the case of relationship between production purpose and psychological factors, it is possible to organize by type of product or targeted persons. Furthermore, if connecting correspondence of psychological factors and design together, it is possible to explain the whole relationship between production purpose and design for each type of product or targeted persons.

\subsection{Design management}

By using psychological factors as mediators, design management using this is considered to be effective for the process of ordering design. So far, when ordering a production, clients had directly included specific design instructions such as layout and coloring in their requests. On the other hand, it may be sufficient to indicate by the psychological factors which are required for the product. In fact, clarifying the relationship between the production purpose and the psychological factors is conducted as market research. Therefore, it is feasible in the process on the client's side to determine what psychological factors will be focused on. On the other hand, designers who receive production orders often know the relationship between psychological factors and design as know-how. For example, in the field of color coordination, Nippon Color \& Design research institute [17], which has spread the scale of "soft-hard "and "cool-warm," has a practical training program for educating designers in which learning the words representing image expressions corresponding to psychological factors and the corresponding colors. It may not be so difficult for designers to express something instructed by the psychological factors in a specific design. It is expected that an efficient production request process can be realized by instructing with psychological factors in the design work. 


\section{Experiment of Design Order Evaluation}

\subsection{Ordering method in crowdsourcing}

Up to this point, it was shown that instructing with psychological factors in design work could lead to the realization of an efficient process of ordering, but in actual crowdsourcing, we confirmed that there is no problem with this method. Using CrowdWorks [10], which is a comprehensive crowdsourcing website in Japan, ordered the actual design job in a competition type and asked the applicants to answer the questionnaire. As a result, we got 5 applicants. Also, the following answers are obtained from the questionnaire.

1) Production purpose is essential and should be more specific.

2) Production purpose is not sufficient.

3) There are two ways: leave everything to the designer or focus the client's image as much as possible.

As for 1), we got opinions such as "It's easy to create if you present objective data such as the target audience, display place, and appeal points". There is an opinion that "There are a wide range of design options, and it is expected that the concrete production purpose will be the criterion when making a concrete design. Because there are so many design options, it is expected that the specific production purpose becomes the criterion when dropping into an actual design." As for 2), we got an opinion: "There is an instruction saying to extract freely from HP and past works, but because it is a work of planners or copywriters, it will be more difficult and leads to decrease of the number of proposals". While production purpose is essential, it seems difficult for normal designers to drop into an actual design without other instructions. Furthermore, we got an interesting opinion as for 3): "The method of leaving it to the designer is an effective way that emphasizes designer's ability and let them present unexpected expressions that the clients never imagine". We also got opinions such as "When leaving it to the designer, the necessary information may be just words," or "When focusing the image of the client, they need to specify the order (importance) of the words to be used, add pictures, colors, other posters to refer to, words to describe the poster image, and give more specific instructions." When leaving to the designer, abstract instructions are required, and when focusing the client's image, more specific instructions are required. In the former case, there is a risk that the designers cannot handle the job depending on their skill, as described in the comment, and in the latter case, the job may be an operating task and reduce designer's creativity.

\subsection{Classification of design ordering method}

Here, we review the ordering method in the job related to design. As a method for ordering a design work, we propose three levels: production purpose, reader's feelings, and concrete design. It will be concrete in this order, but the amount of instructions will also increase. Also, if you give all the instructions at the same time, there is a possibility of conflict. The reader's feelings correspond to psychological factors in the former chapters. In order to simplify the instruction to the minimum required, it may be important at what 
degree of instruction is included in the order. The targeted persons and concept of the product are included in the production purpose. The desired image and the desired color which are prepared on the current crowdsourcing website, are included in each reader's feeling and design.

\subsection{Outline of design order experiment}

We ordered the job related to design to the designers employed by Fuji Xerox group companies in a competition type. Because the designers were distributed in Tokyo, Yokohama, Osaka and Fukushima, they used email and file servers. We used a file server to provide and deliver materials and exchanged the order request and questions via email.

[Competition for production of a volunteer recruitment flyer]

Event: "Fuji Xerox Super Cup Volunteer of inviting children with intellectual disabilities"

Application due date: 15 December (Mon) - 26 December (Fri) 2014

Deliverable: A4 size (PDF)

* A gift voucher will be awarded upon adoption.

This volunteer event is called "Hasu (fraction) Club" and asks participants for the fraction (less than $100 \mathrm{JPY}$ ) of their salaries. An independent volunteer group that conducts activities with the contribution was holding the event. Therefore, it was suitable for this evaluation experiment as it was not subjected to design restrictions by corporate identity (CI). In fact, we called for approximately 30 designers working in the following 8 departments in 7 companies. As a result of recruitment, we got 10 proposals from 7 designers in 5 companies, and the deliverable obtained was satisfactory to a person actually planning events.

\subsection{Details of the experiment method}

In the evaluation experiment, the designers employed in the Fuji Xerox Group companies were ordered with different instructions, and evaluations of the designers about the instructions was compared. This time, the target was not the general crowdsourcing site, but the in-house designers of each group company in order to verify the same product by changing the ordering method. Because different ordering conditions will also be disclosed on a general crowdsourcing site, so it is considered that there is a risk that the contractor will apply only for the competition of the desired conditions and statistical comparison cannot be done. Ordered with different contents that combines three levels of instructions at random; production purpose, reader's feeling, and design. we also considered making 3 groups per each instruction, but because there was also a risk that the number of applicants could not be sufficient, we changed to verify multiple groups with small samples by combining the instructions. The specific instructions of three levels are as follows. 
$<$ Production purpose $>$

- To let many people apply for the event who had not participated in volunteer experience

$<$ Reader's feeling $>$

- Prominent

- Interesting

- Feeling something possible done by own

- Feeling contribution to society

$<$ Design $>$

- Please make the word "volunteer" bigger.

- Please use many photos.

- Please make the color noticeable.

The designer's evaluation of the ordering was measured by a Likert scale (appropriateness, easiness of understanding, specificity, and objectivity). The specific scales are as follows.

\section{Appropriateness}

(5: appropriate. 4: somewhat appropriate, 3 : neither is, 2: somewhat inappropriate, 1: inappropriate)

Easiness of understanding

(5: easy to understand. 4: somewhat easy to understand, 3: neither is, 2: somwhat not easy to understand, 1: not easy to understand,)

\section{Specificity}

(5: specific. 4: somewhat specific, 3: not neither, 2: somewhat abstract, 1: abstract)

\section{Objectivity}

(5: objective. 4: somewhat objective, 3 : not neither, 2 : somewhat subjective, 1: subjective)

\subsection{Result}

12 designers answered the evaluation of the ordering method. An analysis of variance of appropriateness, easiness of understanding, specificity and objectivity was performed depending on whether the order content includes production purpose, reader's feeling and design. As a result, a statistically significant difference was found about appropriateness and easiness of understanding. Firstly, appropriateness was improved $(F(1,8)=6.40, p<0.05)$ when instructing reader's feeling (see Figure 4). 

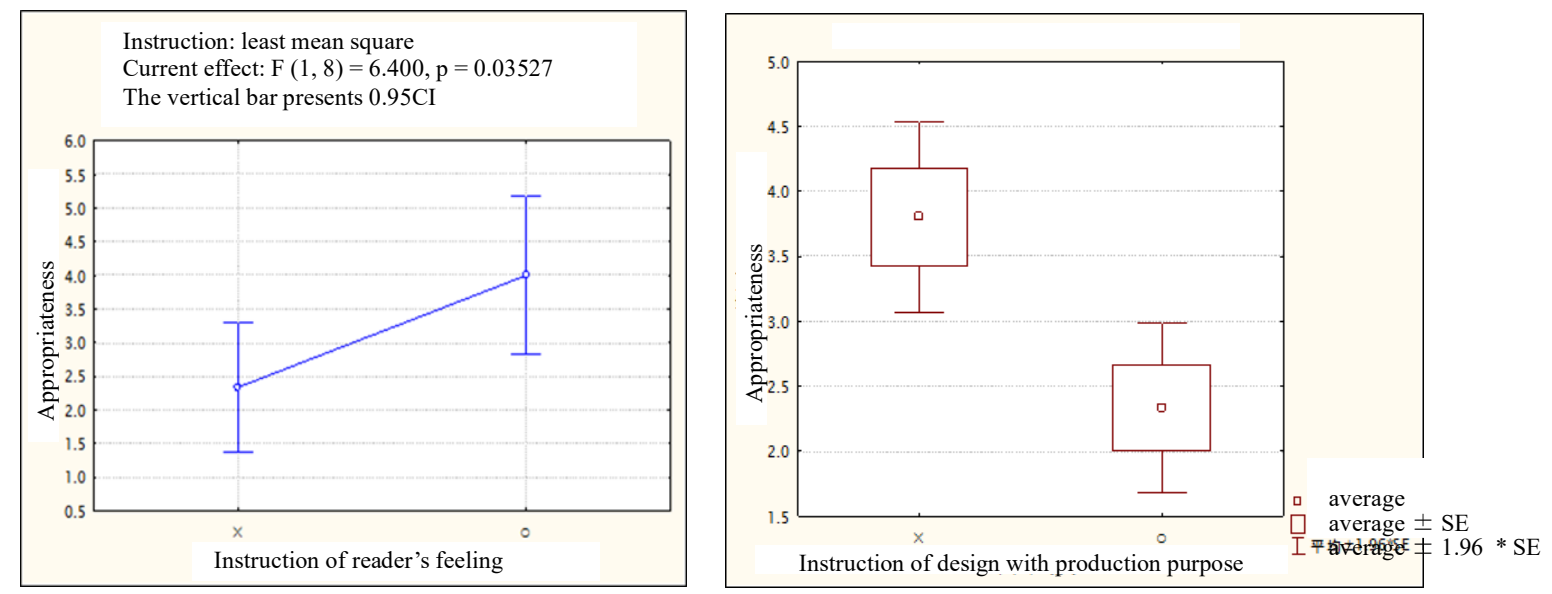

Figure 4: Difference in Appropriateness

with/without instruction of reader's feeling

Next, easiness of understanding was improved $(\mathrm{F}(1,8)=5.88, \mathrm{p}<0.05)$ when instructing reader's feeling, in particular, in the absence of an instruction for production purpose $(\mathrm{F}(1,8)=10.05, \mathrm{p}<0.05)$ (see Figure 5). Herewith, it is considered that reader's feeling can complement production purpose in terms of easiness of understanding. Appropriateness decreased $(\mathrm{t}=2.64, \mathrm{f}=6, \mathrm{p}<0.05)$ when instructing production purpose and design at the same time (see Figure 4). The cause of this is imagined at the moment, but it is thought that the design instruction may contradict the production purpose. The design instruction approaches the mere operation instruction, and thus may reduce the motivation to create. It may be noticeable when the contradiction is felt between the instruction of design and production purpose.
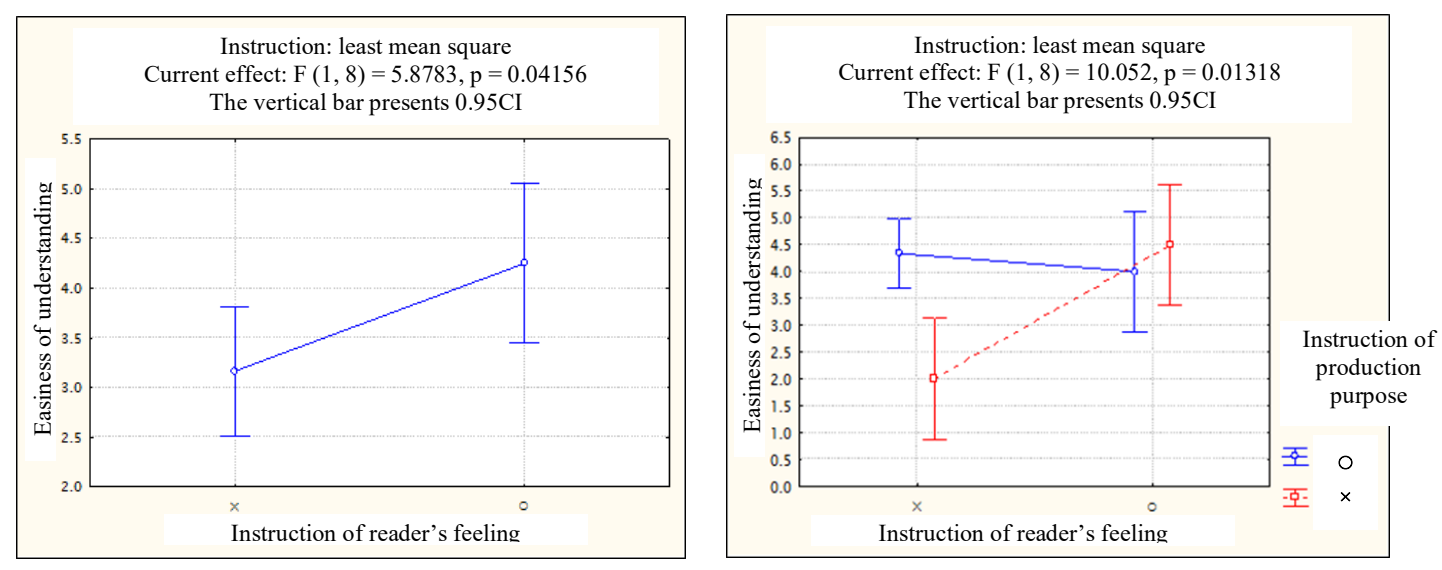

Figure 5: Difference in Easiness of understanding with/without instruction of reader's feeling

With regard to specificity and objectivity, no statistically significant difference was found at this time.

The designer's questions for confirmation that were exchanged from ordering to application were classified into three types: concept image, material usage, and output form. A group whose order contents did not include reader's feeling asked a question about concept image, 
but no other group asked about this. The following comment was given from the designer who asked the question about concept image.

"I would like to know the details of the presentation of the planning concept, themes and requests, and the target group (who should I appeal to?)."

"Isn't this method difficult for design proposals as a solution to the vague needs and requirements of the client?"

"I would like you to describe in more detail the part of the person who has no experience as a production purpose volunteer."

On the other hand, questions regarding material usage and output form were the same from all groups. In this experiment, because it was a competition type, there was no precontractual interaction like the project type, and the absolute number of questions was small, so statistical verification could not be performed. However, if the contents of the order include reader's feeling, it may be possible to reduce the number of questions asked by the designer for confirmation, especially regarding the concept image. In addition, this time, 2 designers have made multiple proposals. Both were designers of groups whose order contents did not include reader's feeling. Even if the ordering content includes the production purpose but does not include the reader's feeling, it is possible to assume that multiple images are produced, so it can be imagined that multiple proposals have been made in advance. Since this time it was a competition type, it is not a bad thing that many proposals gather, but in the case of a project type, it is effective to include reader's feeling in the order contents in order to reduce the effort of the contractor. From the results, it was not possible to statistically verify that the number of questions of the designer for confirmation decreases when the order is placed in the reader's feeling. However, there is such a tendency, and it cannot be denied.

\section{Conclusion}

\subsection{Results and discussion}

We presented that there are three levels; production purpose, reader's feeling and specific design concerning the ordering method of the jobs related to design. Among these, by ordering including reader's feeling, it was expected that the difference in the image of the deliverables of the client and the worker would be small, and the worker's evaluation on the order method would be high. In this regard, it was statistically confirmed that the appropriateness and the easiness of understanding improve when the order content includes reader's feeling. In particular, in the absence of an instruction for production purpose, the instruction of reader's feeling helps to enhance easiness of understanding. Herewith, it is considered that the inclusion of reader's feeling in the order has enhanced the worker's evaluation on the ordering method.

On the other hand, it is not yet known how the client's evaluation differs depending on the difference in the ordering method. We consider that the survey results of aforementioned "Promotion program of utilizing crowdsourcing at The Small and Medium Enterprise Agency 2016" [11] will become a good reference. In this ordering experience program, 
the crowdsourcing producer was supposed to support the definition of requirements at the time of ordering from the following viewpoints.

- Clarify and specifically describe the purpose and outline of the production of the flyer with using templates.

- Specify the desired image, etc. with using U/I to tell the worker the image of the deliverable.

The former corresponds to production purpose and the latter corresponds to reader's feeling. Herewith, it is considered that if the crowdsourcing producer supports the definition of requirements from these viewpoints, the number of applicants and proposals might increase as the worker accepted it well. Further, $77 \%$ of the respondents claimed "placing order / defining requirement" as the part that they asked crowdsourcing producers for help. It was the highest ratio among other particulars such as "member registration (41\%)", "correction request (32\%)", "selection of worker $(40 \%)$ ", and "inspection of deliverable $(16 \%)$.

\subsection{Limitations}

The verified jobs were limited to the jobs related to design at this time. The characteristic is that the readers decide whether the production purpose can be achieved or not, and it is not by the client or the worker. For this reason, this survey does not mention the quality of the deliverables. The results may not be applicable to regular operations such as development or desk work that are different from design work. However, we consider that there is a possibility applied to the business of producing products that depend on customer's mind such as marketing.

\subsection{Future perspective}

With regard to the worker's evaluation, we examined what causes the satisfaction level of the client, based on the survey result of "Promotion program of utilizing crowdsourcing at The Small and Medium Enterprise Agency 2016" [11]. We asked people with actual ordering experiences whether the definition of requirements was successful or not. The result showed that the number of applicants and proposals increase and the satisfaction level of the client is enhanced as the definition is written successfully. From the contents of seminar of the ordering experience program, it is expected that the requirements have defined more successfully as the order includes more production purpose or reader's feeling. In order to clarify this, it seems to be necessary to individually confirm what kind of instructions the client has given. We intend to present the possibility of simplifying the instruction and enhancing worker's evaluation through ordering with reader's feeling. Also, the number of applicants and proposals will increase and the orders will be more effective ordering for the clients. In addition, we have not directly mentioned the improvement of the order acceptance ratio and avoidance of problems which are the current issues of crowdsourcing, but we hope that the results in this study will contribute to these issues. 


\section{References}

[1] Fuji Xerox Co., Ltd., Dec. 2014; http://www.fujixerox.co.jp/solution/gs/.

[2] J. Howe, "The Rise of Crowdsourcing," Dec. 2014; http://archive.wired.com/wired/archive/14.06/crowds.html?pg=4\&topic=crowds.

[3] “A Survey on Usage of Crowdsourcing in Japan," Y's Staff Co., Ltd. report, Small and Medium Enterprise Agency, 2013 (in Japanese).

[4] A. Kittur, B. Smus, and S. Khamkar, R. E. Kraut, "Crowdforge: Crowdsourcing complex work," Proceedings of the 24th annual ACM symposium on User interface software and technology, 2011, pp.43-52.

[5] D. Chandler, and A. Kapelner, "Breaking monotony with meaning: Motivation in crowdsourcing markets," Journal of Economic Behavior \& Organization 90, 2013, pp.123-133.

[6] C. Eickhoff, and A. P. d. Vries, "Increasing cheat robustness of crowdsourcing tasks," Information retrieval 16(2), 2013, pp.121-137.

[7] A. Kittur, E. H. Chi, and B. Suh, "Crowdsourcing user studies with Mechanical Turk," Proceedings of the SIGCHI conference on human factors in computing systems, 2008, pp.453-456.

[8] H. Jeffrey, and M. Bostock, "Crowdsourcing graphical perception: using mechanical turk to assess visualization design," Proceedings of the SIGCHI Conference on Human Factors in Computing Systems, 2010, pp.203-212.

[9] Lancers Inc., May. 2020; http://www.lancers.jp/.

[10] CrowdWorks Inc., May. 2020; http://crowdworks.co.jp/.

[11] Announcement of "Cloud Sourcing Ordering Experience" for SMEs developed by the Consortium for Promoting Crowdsourcing Utilization, " May. 2020; https://www.japan-telework.or.jp/oshirase/75.html.

[12] N.P. Suh, “Axiomatic Design of Mechanical Systems," Journal of Mechanical Design and the journal of Vibration and Acoustics, Transactions of the ASME, Volume 117, June 1995, pp.1-10.

[13] Yoshiyuki Matsuoka, "Multispace Design Model: Toward the Construction of the Framework for Design Science," The 55th Spring Seminar of Japanese Design Society Conference summary collection, 2008 (in Japanese).

[14] H. Yanagisawa, T. Murakami, K. Ohtomi, and R. Hosaka, “Quantification method of product emotional quality considering its diversity (application to quantification of emotional quality in product sound design)," Transactions of the Japan Society of Mechanical Engineers, Series C, Vol.74, No.746, 2008, pp.2607-2616 (in Japanese). 
[15] Takeshi Tanaka, Masakazu Fujimoto, Tomomi Urushihara, “On the evaluation scale for document expression quality," Proceedings of the 42nd National Conference of Information Processing Society of Japan, the first half of 1991 (3), 1991, pp. 331-332 (in Japanese).

[16] R. E. Petty, J. T. Cacioppo, and D. Schumann, “Central and peripheral routes to advertising effectiveness: The moderating role of involvement," Journal of consumer research, 10, 1983, pp. 135-146.

[17] "What is the image scale | NCD-WEB | Japan Color Design Research Institute for Color Psychology, Consumer Research, Trend Analysis," Japan Color Design Research Institute Inc., May. 2020;

http://www.ncd-ri.co.jp/about/image_system/imagescale.html. 